\title{
Is it taking longer to die in paediatric intensive care in England and Wales?
}

\author{
Adrian Plunkett, ${ }^{1}$ Roger C Parslow ${ }^{2}$
}

\begin{abstract}
${ }^{1}$ Paediatric Intensive Care Unit, Birmingham Children's Hospital, Birmingham, UK ${ }^{2}$ Leeds Institute of Cardiovascular and Metabolic Medicine, University of Leeds, Leeds, UK
\end{abstract}

\section{Correspondence to} Dr Roger C Parslow, Leeds Institute of Cardiovascular and Metabolic Medicine, Room 8.49, Worsley Building, Clarendon Way, University of Leeds, Leeds LS2 9JT, UK; r.c.parslow@leeds.ac.uk

Received 18 August 2015 Revised 14 January 2016 Accepted 16 February 2016 Published Online First 7 March 2016

\section{SLinked}

- http://dx.doi.org/10.1136/ archdischild-2016-310637

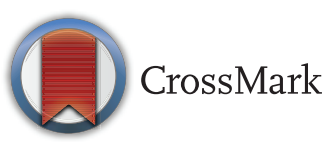

To cite: Plunkett $A$ Parslow RC. Arch Dis Child 2016;101:798-802.

\begin{abstract}
Introduction All-cause infant and childhood mortality has decreased in the UK over the last 30 years. Advances in paediatric critical care have increased survival in paediatric intensive care units (PICUs) but may have affected how and when children die in PICU. We explored factors affecting length of stay (LOS) of children who died in PICU over an 11-year period. Methods We analysed demographic and clinical data of 165473 admissions to PICUs in England and Wales, from January 2003 to December 2013. We assessed time trends in LOS for survivors and non-survivors and explored the effect of demographic and clinical characteristics on LOS for non-survivors.
\end{abstract}

Results LOS increased 0.310 days per year in nonsurvivors $(95 \% \mathrm{Cl} 0.169$ to 0.449$)$ and 0.064 days per year in survivors $(95 \% \mathrm{Cl} 0.046$ to 0.083$)$. The proportion of early deaths ( $<24 \mathrm{~h}$ of admission) fell $0.44 \%$ points per year $(95 \% \mathrm{Cl}-0.971$ to 0.094$)$, but the proportion of late deaths ( $>28$ days of PICU stay) increased by $0.44 \%$ points per year $(95 \% \mathrm{Cl} 0.185$ to $0.691)$. The paediatric index of mortality score in early deaths increased by $0.77 \%$ points per year $(95 \% \mathrm{Cl}$ $0.31 \%$ to $1.23 \%$ ).

Discussion Increased LOS in children who die in PICU is driven by a decreased proportion of early deaths and an increased proportion of late deaths. This trend, combined with an increase in the severity of illness in early deaths, is consistent with a reduction in early mortality for acutely ill children, but a prolongation of life for those children admitted to PICU with life-limiting illnesses.

\section{INTRODUCTION}

The rate of childhood mortality from all causes in the UK has decreased over the last three decades. ${ }^{1}$ Data from the UK Paediatric Intensive Care Audit Network (PICANet) show that crude mortality in British paediatric intensive care units (PICUs) has followed the national trend and fallen consistently from $5.5 \%$ in $2003 / 2004$ to $3.7 \%$ in $2013 .^{2}{ }^{3}$ This fall in mortality has occurred during a period of considerable growth in the demand and capacity of PICU (admissions to British PICUs from 2004 to 2013 have risen by $15 \%) .^{3}$

While survival is improving, the prevalence of life-limiting illness and chronic disease in British children is increasing. ${ }^{4}$ Previous research from the USA has shown that children with complex chronic conditions (CCCs) have a higher mortality and experience longer stays in hospital prior to death when compared with children without CCC. ${ }^{5}$ The majority (two-thirds) of deaths in British children occur in children with chronic health conditions. ${ }^{1}$

\section{What is already known on this topic}

- Childhood mortality in the UK is decreasing

- The majority of childhood deaths in the UK occur in children with chronic health conditions.

- The prevalence of life-limiting conditions in children in the UK is increasing.

\section{What this study adds}

- The length of stay of children who die in a paediatric intensive care unit is increasing.

- The scenario of an early death in paediatric intensive care unit (PICU) following treatment failure is being replaced by the death of a child at the end of a long PICU admission.

Excluding deaths due to injury, the majority of childhood deaths in the UK occur in hospital ${ }^{1}$ and the most common location for childhood hospital death is the PICU. ${ }^{6}$

The changing epidemiology of death and disability in children, along with improved survival from intensive care, suggests that the characteristics of the children who die in PICU may also be changing over time.

We investigated whether there is a significant increase in the length of time that children spend in a PICU before their death and, if this is the case, whether this effect is universal or confined to a specific subpopulation of patients. We also analysed trends in PICU bed-day utilisation among children who die in PICU.

\section{METHODS}

We analysed demographic and clinical data submitted to PICANet of all children admitted to PICUs in England and Wales between January 2003 and December. PICANet collects demographic and clinical information of all children admitted to PICUs in the UK and the Republic of Ireland, and this is complete for England and Wales for all years from 2003 onwards. Details of data collection and validation methods are published in the PICANet Annual Report. ${ }^{2}$ We analysed three specific aspects of the data: overall trends in length of stay (LOS) by vital status, trends in LOS for early and late deaths, bed utilisation and clinical and demographic factors that may explain these trends. We further 
examined the relationship between clinical and demographic factors and trends in LOS.

\section{Trends in LOS in survivors and non-survivors}

Mean and median LOS in days was plotted against individual year for children who had died in PICU and those who had survived to PICU discharge. Linear regression was used to assess the relationship between LOS and year of admission. The following variables were analysed to establish whether LOS for children who die in PICU was different in different sections of the PICU population: diagnostic group, sex, South Asian ethnicity (based on two name recognition algorithms) ${ }^{78}$ and an areabased measure of deprivation (Index of Material Deprivation 2010) ${ }^{9}$ derived from the children's address on admission. We also used the expected probability of mortality calculated using the paediatric index of mortality (PIM) ${ }^{10}$ using recalibrated coefficients calculated by Brady et $a l^{11}$ and supplied by the authors as a continuous variable and in predefined expected probability of death (POD) groups $(<1 \% ; 1 \%$ to $<5 \% ; 5 \%$ to $<15 \% ; 15 \%$ to $<30 \%$ and $30 \%+)$. This recalibrated version of PIM was used because data collection for PIM2 did not take place throughout the study period, but data for PIM were available. We also analysed LOS over time by the broad diagnostic groups reported by PICANet. Analyses of the children's individual characteristics used random-effects linear regression to take account of centre (PICU) effects by using a random intercept term, and the natural log transformation of LOS (LOGLOS) to account for the skewed nature of the LOS data. Likelihood ratio (LR) tests, Akaike's information criterion (AIC) and Bayesian information criterion (BIC) were used to assess the improvement in fit of models when an interaction term was introduced to model the relationship between these individual characteristics and year of admission.

\section{Analysis of early and late deaths}

Further analysis was undertaken by stratifying deaths by LOS to allow comparison of early deaths $(<1$ day in PICU) and late deaths ( $>28$ days in PICU). For this purpose, the following predefined LOS strata were used: 1 (early deaths): <1 day; 2:1-3 days; 3:3-7 days; 4:7-14 days; 5:14-28 days; 6 (late deaths): >28 days. For each stratum, linear regression and random-effects linear regression analysis was performed to describe temporal trends in LOS. Differences in the characteristics of early and late deaths over the time period were further analysed by comparison of mean expected POD estimated by PIM score expressed as a percentage, as a surrogate for severity of illness on PICU admission.

\section{Bed-day utilisation}

Trends in bed-day utilisation were determined by calculating the sum of PICU bed-days for survivors and non-survivors for each year of the time period, aggregating bed-days for those children who had been admitted on more than one occasion. Regression results are presented with 95\% CIs. All statistical analyses were carried out in Stata Release V.12 (StataCorp. 2011. Stata Statistical Software: Release V.12. College Station, TX).

\section{RESULTS}

Results are based on 165473 admissions representing 112360 children of whom 157918 were discharged alive and 7555 died in PICU. Linear regression analysis of trends in LOS showed a significant trend for increased LOS in non-survivors (increased LOS of 0.314 days per year, $95 \%$ CI 0.174 to 0.454 ) compared with survivors (increase in LOS of 0.064 days per year, 95\% CI
0.046 to 0.083$)$. The random-effects model estimates were 0.310 (95\% CI 0.169 to 0.449 ) and 0.064 (95\% CI 0.046 to 0.083 ) days per year, respectively, reflecting a marginal effect of the variability between PICUs (mean change in LOS varied from -5 to +6 days per year). Mean and median LOS in days are plotted by year of admission in figure 1 .

Linear regression analysis showed a statistically non-significant fall in the proportion of early deaths over the study period of $0.44 \%$ points per year in children dying in the first day $(95 \%$ CI -0.971 to 0.094 ) and a statistically significant rise in the proportion of late deaths of $0.44 \%$ points per year in those children dying who stay $>28$ days (95\% CI 0.185 to 0.691 ). The respective estimates from the random-effects regression were $-0.15 \%$ points per year $(95 \% \mathrm{CI}-0.770$ to 0.471$)$ and $0.37 \%$ points per year $(95 \%$ CI 0.111 to 0.621$)$. The attenuation in the estimate for the proportion of children dying in the first day was attributable to four PICUs with very low or no mortality. The percentage of deaths over time in those who died within one day and after 28 days are shown in figure 2, together with the number of deaths in each year.

Further analysis of the early versus late deaths demonstrated a temporal trend of increasing PIM score in the early deaths of $0.77 \%$ points per year (95\% CI $0.31 \%$ to $1.23 \%)$. No significant temporal trend was present in the PIM score in the late deaths group (figure 3). There was a statistically significant improvement in model fit when an interaction term between PIM POD group and year of admission was included (LR test $\left.\chi^{2}, 10.71, p=0.0301\right)$. The AIC and BIC confirmed this improvement.

Analysis of bed-day utilisation (summed over all patients, survivors and non-survivors) demonstrated a rise in total bed-day use in survivors, but no significant change in the overall bed-day use in non-survivors (figure 4).

Regression analysis of diagnostic group revealed no specific diagnosis driving the increase in LOS (data not shown). Likelihood ratio tests for improvement in the random-effects regression model fit by including an interaction term between

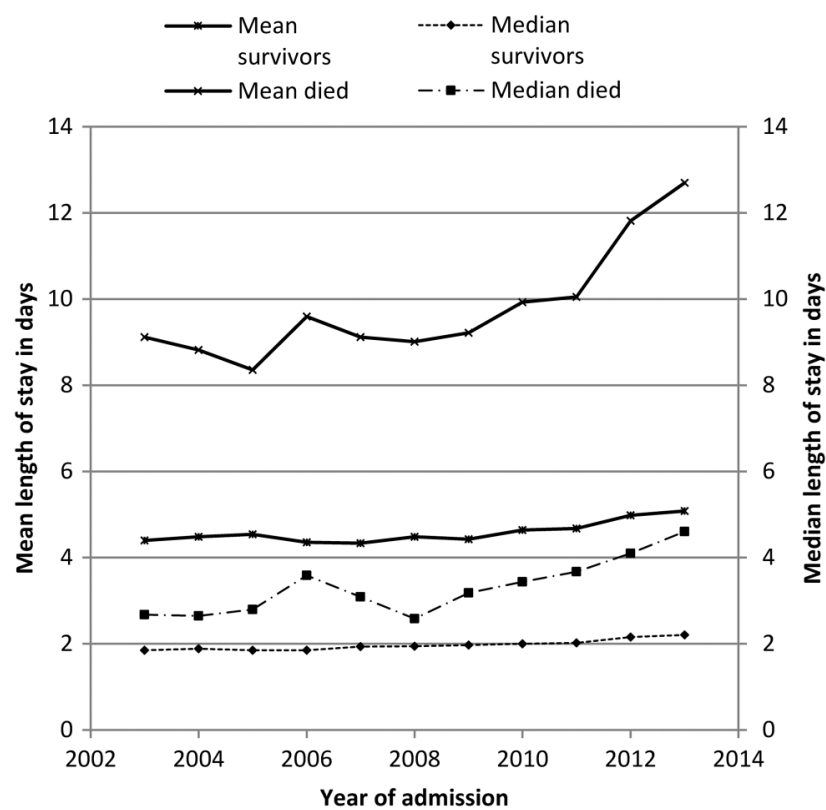

Figure 1 Mean and median length of stay in days for survivors and non-survivors admitted to a paediatric intensive care unit in England and Wales between January 2002 and December 2013. 


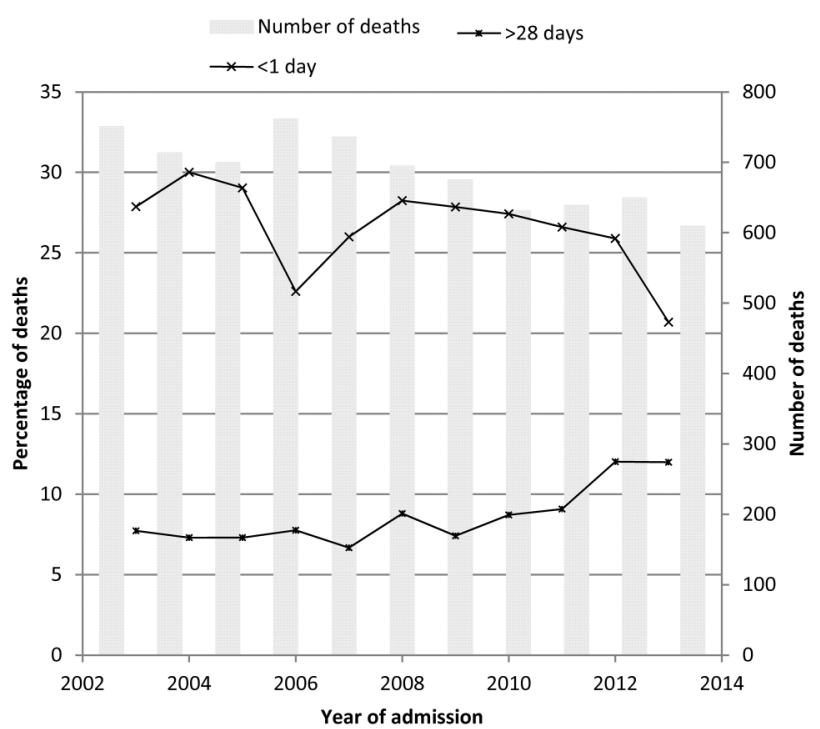

Figure 2 Percentage of deaths occurring early $(<1$ day paediatric intensive care unit (PICU) length of stay (LOS)) and late ( $>28$ days PICU LOS) over time.

year of admission and sex, South Asian ethnicity and deprivation respectively revealed that none of these variables had a significant impact on the observed increase in LOS of those children who died in PICU.

\section{DISCUSSION}

The LOS of children who die in PICU is increasing over time. More children who previously would have died early in PICU admission are now surviving, but some of this group will die later in the same PICU admission. There is a trend of rising severity of illness on admission (using expected probability of mortality estimated by PIM as a proxy) in children who die within $24 \mathrm{~h}$ of admission to PICU. Together with the falling mortality rates in PICUs, these trends are consistent with changes in healthcare delivery, which result in increased survival

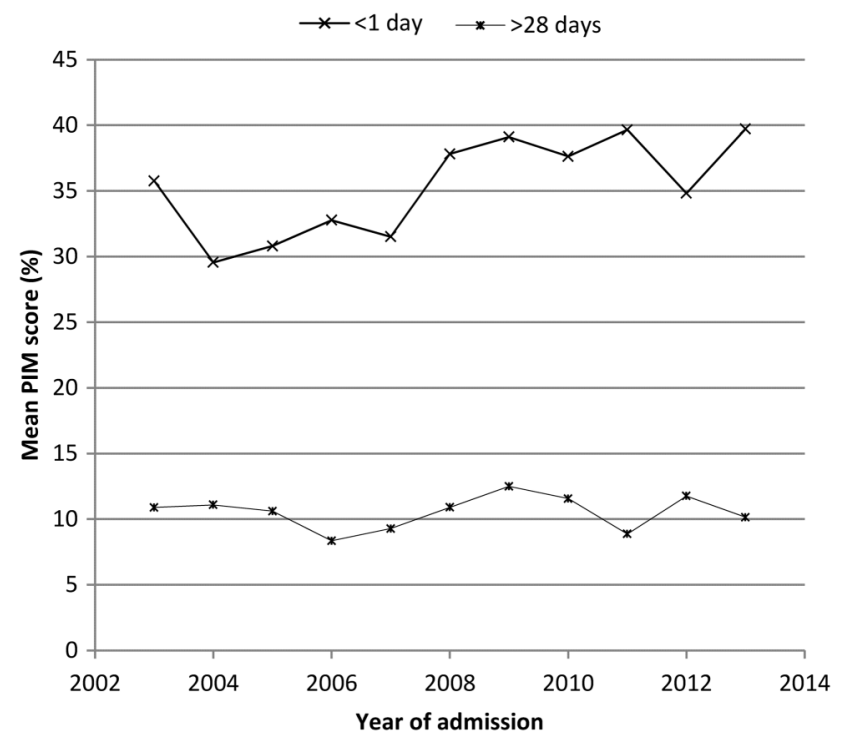

Figure 3 Mean paediatric index of mortality (PIM) (\%) score of non-survivors over time, stratified by early $(<1$ day) versus late (>28 days) deaths.

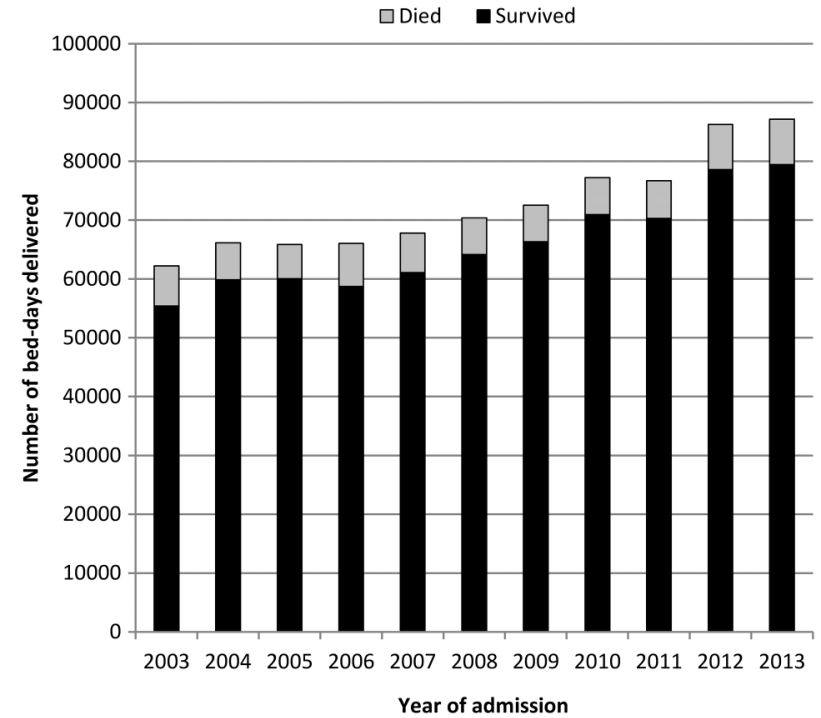

Figure 4 Summed bed-day utilisation over time for survivors and non-survivors.

of children in the acute phase of critical illness. ${ }^{12}$ Advances in paediatric medicine, including the introduction of acute care bundles, development of specialist training programmes, improved perioperative care, organisation of PICUs and the development of retrieval medicine for critically ill children, may have contributed to this trend of increased survival. ${ }^{12-15}$ Additional factors may contribute to the lengthening of terminal admissions in certain patient subgroups, including changes in patient and public expectations of healthcare, an increase in shared decision-making and a fear of litigation among physicians when considering end-of-life decisions for children. ${ }^{16}$ Within the limits of this observational study, it is not possible to assess the contribution of each of these factors to the trends we have identified, and further research in this area is indicated.

The increasing bed-day utilisation among children who survive PICU admission reflects the growth of the national capacity of PICUs during the study period. The bed-day utilisation among non-survivors is not increasing, despite a significant increase in LOS in this group. This can be explained by the falling mortality rate during the study period. Thus, although the average LOS among children who die is increased, they represent fewer of the PICU admissions.

Our study indicates that children who would previously have died early in PICU are now living longer. It is likely that some of these children are surviving to discharge from PICU, and some may be surviving with chronic disease. Thus, the rising prevalence of life-limiting condition (LLC) and chronic conditions in children may represent one of the costs of improved survival from PICU. This association has been described elsewhere: a cohort study of outcomes following PICU admission in a single PICU in Melbourne, spanning three decades, showed a falling mortality rate accompanied by a rising disability rate among PICU survivors. ${ }^{17}$ Studies from other international centres also show a rising prevalence of CCC in US hospitalisations, ${ }^{5} 18$ and in PICU admissions in France, ${ }^{19}$ Greece $^{20}$ and the USA. $^{21}$ The characteristics of patients in PICU are therefore changing over time as children with chronic illness experience higher rates of hospitalisation, PICU admission and PICU readmission, longer LOS and higher mortality risk. ${ }^{5} 2223$

These studies capture important epidemiological trends and highlight the lack of consistent definitions of chronic illness and 
disability. LLC and CCC describe similar, but not mutually inclusive, clinical states. ${ }^{4} 24$

The general trend of increased LOS in non-survivors was not explained by differences between diagnostic group, socioeconomic status, age, sex and South Asian ethnicity.

This study highlights several areas worthy of further research. Follow-up data on patients who survive PICU in the UK are rare and lacking detail. Longitudinal follow-up studies examining the long-term survival, educational level, quality of life and comorbidities of PICU survivors in the UK are required to provide a better understanding of the health costs of improved PICU survival.

Further research is also warranted into the ethical issues surrounding the death of a child and their effects on patients, families and staff. The scenario of an early death in PICU following treatment failure is being replaced by the death of a child at the end of a long PICU admission. The mode of these later deaths is usually the result of planned withdrawal of life support; observational data from studies in single centres elsewhere support the view that this mode of death is increasingly common in PICU. ${ }^{25} 26$ These findings indicate that the roles of palliative care teams, the use of advanced care planning (ACP) and multidisciplinary working in the clinical management of children with LLC/CCC will be areas of future service development and research. Existing evidence points to variability in practice in this area: a single-centre study examining timing of death following agreement for withdrawal of life support in PICU showed wide variety of timings, ${ }^{25}$ and a recent survey of British PICUs found that there is wide variability in documentation for ACP across the UK. ${ }^{27}$ The rising prevalence of LLC/ CCC in the childhood population, coupled with our finding that children who die in PICU are spending a longer time in the PICU prior to death, emphasises the need to gain a better understanding of end-of-life care for children. In particular, a key area for future research is to focus on understanding the risks and benefits of ACP.

Limitations of our study include a lack of information on mode of death (such as withdrawal of life support or failure of treatment) and the use of ACP prior to admission or during admission to PICU and the requirement for PICUs contributing to PICANet to only code a primary reason for the admission with the coding of comorbidity requested but not mandated. This makes a robust analysis of diagnostic subgroups that rely on good coding depth unfeasible, and we have avoided such an analysis as it may have introduced bias. These coding issues also limit the ability to infer prevalence of LLCs or CCCs in children. As the PICANet data set comprises the whole population of children admitted to PICUs, there is no sample selection bias. Rigorous validation at data entry and post hoc validation reporting and processing ensure missing data are rare and the variables used in our analysis have an extremely high level of completion. $^{3}$

\section{Twitter Follow Adrian Plunkett at @adrianplunkett}

Acknowledgements The authors thank all the staff in participating hospitals who have collected data for PICANet. A list of participating organisations is given at: http://www.picanet.org.uk/About/Participating-Organisations/. The authors are also grateful to the UK Paediatric Intensive Care Society for continued support and to the members of the PICANet Steering Group and Clinical Advisory Group.

Contributors AP conceived the hypothesis, conducted the literature review, and contributed to data interpretation and wrote the main body of the manuscript. AP and RCP jointly developed the analysis plan. RCP analysed the data, contributed to data interpretation and edited the text of the manuscript. RCP is the guarantor. RCP affirms that the manuscript is an honest, accurate and transparent account of the study being reported; that no important aspects of the study have been omitted; and that any discrepancies from the study as planned have been explained. RCP had full access to all of the data in the study and had final responsibility for the decision to submit for publication.

Funding The PICANet Audit is commissioned by the Healthcare Quality Improvement Partnership (HQIP) as part of the National Clinical Audit Programme (NCA). HQIP is led by a consortium of the Academy of Medical Royal Colleges, the Royal College of Nursing and National Voices. Its aim is to promote quality improvement and, in particular, to increase the impact that clinical audit has on healthcare quality in England and Wales. HQIP holds the contract to manage and develop the NCA Programme, comprising more than 30 clinical audits that cover care provided to people with a wide range of medical, surgical and mental health conditions. The PICANet Audit is funded by NHS England, the Welsh Government, NHS Lothian/ National Service Division NHS Scotland, the Royal Belfast Hospital for Sick Children, The National Office of Clinical Audit (NOCA), Republic of Ireland and HCA Healthcare. The sponsor of the study had no role in the study design, data collection, analysis or interpretation of the findings and no role in the writing of this report.

Competing interests None declared.

Ethics approval Trent Medical Research Ethics Committee, ref. 05/MRE04/17 +5.

Provenance and peer review Not commissioned; externally peer reviewed.

Data sharing statement The data for this study are available in raw form from PICANet. Aggregate data and unpublished analysis tables are available from the authors.

\section{REFERENCES}

1 Child Health Reviews UK, Clinical Outcome Review Programme. Overview of Child Deaths in the 4 UK countries. September 2013. http://www.rcpch.ac.uk/ child-health-reviews-uk/programme-findings/programme-findings

2 Davey N, Draper ES, Jones S, et al. Paediatric Intensive Care Audit Network Annual Report, March 2003-February 2004. Universities of Leeds, Leicester and Sheffield, 2004:1-94. http://www.picanet.org.uk/Audit/Annual-Reporting/Annual-ReportArchive/PICANet_National_Report_2004.pdf (accessed 27 Feb 2015).

3 Paediatric Intensive Care Audit Network. A Decade of Data (published September 2014): Universities of Leeds and Leicester. http://www.picanet.org.uk/Audit/ Annual-Reporting/PICANet_A_Decade_of_Data_2014_Annual_Report_Summary. pdf (accessed 27 Feb 2015).

4 Fraser LK, Miller M, Hain R, et al. Rising national prevalence of life-limiting conditions in children in England. Pediatrics 2012;129:e923-9.

5 Simon TD, Berry J, Feudtner $C$, et al. Children with complex chronic conditions in inpatient hospital settings in the United States. Pediatrics 2010;126:647-55.

6 Ramnarayan P, Craig F, Petros A, et al. Characteristics of deaths occurring in hospitalised children: changing trends. J Med Ethics 2007;33:255-60.

7 Nanchahal K, Mangtani P, Alston M, et al. Development and validation of a computerized South Asian Names and Group recognition Algorithm (SANGRA) for use in British health-related Studies. J Public Health Med 2001;23:278-85.

8 Bradford Health Authority and City of Bradford Metropolitan District Council. Nam Pehchan: a program to identify and analyse South Asian names version 2.1. City of Bradford Metropolitan District Council, 2002.

9 Department for Communities and Local Government. English Indices of Deprivation, 2010 (Data file). https://www.gov.uk/government/uploads/system/uploads/ attachment_data/file/6872/1871524.xls (accessed 17 Dec 2014).

10 Shann F, Pearson G, Slater A, et al. Paediatric index of mortality (PIM): a mortality prediction model for children in intensive care. Intensive Care Med 1997;23:201-7.

11 Brady A, Harrison D, Black S, et al., on behalf of the UK PICOS Study Group. Assessment and optimisation of mortality prediction tools for admissions to paediatric intensive care in the United Kingdom. Pediatrics 2006;117:733-42.

12 Carcillo JA. What's new in pediatric intensive care. Crit Care Med 2006;34:S183-90.

13 Booy R, Habibi $P$, Nadel $S$, et al. Reduction in case fatality rate from meningococcal disease associated with improved healthcare delivery. Arch Dis Child 2001;85:386-90.

14 Pearson G, Shann F, Barry P, et al. Should paediatric intensive care be centralised? Trent versus Victoria. Lancet 1997;349:1213-17.

15 Ramnarayan $\mathrm{P}$, Thiru K, Parslow RC, et al. Effect of specialist retrieval teams on outcomes in children admitted to paediatric intensive care units in England and Wales: a retrospective cohort study. Lancet 2010;376:698-704.

16 Ballard DW, Li Y, Evans J, et al. Fear of litigation may increase resuscitation of infants born near the limits of viability. J Pediatr 2002;140:713-18.

17 Namachivayam $P$, Shann F, Shekerdemian $L$, et al. Three decades of pediatric intensive care: who was admitted, what happened in intensive care, and what happened afterward. Pediatr Crit Care Med 2010;11:549-55.

18 Burns $\mathrm{KH}$, Casey PH, Lyle RE, et al. Increasing prevalence of medically complex children in US hospitals. Pediatrics 2010;126:638-46.

19 Cremer R, Leclerc F, Lacroix J, et al., GFRUP/RMEF Chronic Diseases in PICU Study Group. Children with chronic conditions in pediatric intensive care units located in predominantly French-speaking regions: prevalence and implications on rehabilitation care need and utilization. Crit Care Med 2009;37:1456-62. 
20 Briassoulis G, Filippou O, Natsi L, et al. Acute and chronic paediatric intensive care patients: current trends and perspectives on resource utilization. QJM 2004;97:507-18.

21 Edwards JD, Houtrow AJ, Vasilevskis EE, et al. Chronic conditions among children admitted to U.S. pediatric intensive care units: their prevalence and impact on risk for mortality and prolonged length of stay* . Crit Care Med 2012;40:2196-203.

22 Berry JG, Hall M, Hall DE, et al. Inpatient growth and resource use in 28 children's hospitals: a longitudinal, multi-institutional study. JAMA Pediatr 2013;167:170-7.

23 Feudtner C, Christakis DA, Zimmerman FJ, et al. Characteristics of deaths occurring in children's hospitals: implications for supportive care services. Pediatrics 2002;109:887-93.
24 Feudtner C, Christakis DA, Connell FA. Pediatric deaths attributable to complex chronic conditions: a population-based study of Washington state, 1980-1997. Pediatrics 2000;106(Pt 1):205-9.

25 Oberender F, Tibballs J. Withdrawal of life-support in paediatric intensive care-a study of time intervals between discussion, decision and death. BMC Pediatr 2011;11:39.

26 Sands R, Manning JC, Vyas $\mathrm{H}$, et al. Characteristics of deaths in paediatric intensive care: a 10-year study. Nurs Crit Care 2009;14:235-40.

27 Mitchell S, Plunkett A, Dale J. Use of formal advance care planning documents: a national survey of UK Paediatric Intensive Care Units. Arch Dis Child 2014;99:327-30

\section{ARCHIVIST}

\section{Can early allergen exposure prevent food allergy?}

The controversy continues over whether children can be protected from developing food allergies by deliberately giving them foods to which they might be allergic in the early months. Most official guidance continues to recommend delayed introduction, although evidence suggesting benefit from doing the opposite is accumulating. Recently the LEAP trial suggested that early peanut exposure is protective in atopic children (Du Toit G et al. NEJM 2016. doi:10.1056/NEJMoa1414850). But what about other foods?

A London-based group designed a trial to try and answer this (EAT Trial: Perkins M, et al. NEJM 2016; doi:10.1056/NEJMoa1514210). They recruited over 1300 exclusively breast-fed infants from the UK population at 3 months of age, not looking specifically for those at high risk of allergy. They were randomised to either early introduction of peanut, egg, cow's milk, sesame, white fish and wheat, or to the standard UK practice of avoiding all these foods until after 6 months. The primary outcome was an allergic reaction following formal food challenge between 1 and 3 years.

The results were not straightforward. There was a high prevalence of deviation from the study protocol, particularly amongst the early-introduction group, and only around 40\% strictly adhered. They therefore decided to analyse their results in two ways: per-protocol (PP) including only those who adhered to the protocol; and intention-to-treat (ITT) which includes all those with usable data whether or not they adhered, and thus probably more closely resembles what would happen 'in real life'.

Both analyses showed lower rates of allergy in the early introduction group, but in the ITT analysis it was not statistically significant for any of the food groups (overall 5.6\% vs. 7.1\%; $\mathrm{p}=0.32$ ). However in the PP analysis the difference was significant (overall 2.4\% vs. 7.3\%; $\mathrm{p}=0.01)$. Early introduction appeared particularly protective for peanut as in the LEAP trial ( $0 \%$ vs $2.5 \%)$ and egg $(1.4 \%$ vs $5.5 \%$ ) but not for the other foods. Skin prick tests, done in some, seemed to show the same trend.

Importantly, they found that early introduction was safe, with no serious allergic reactions requiring adrenaline. They also noted a 'dose-dependent' response for peanut and egg, with those who ate more being less likely to develop allergy.

An editorial by Gary Wong reflects on the difficulties in doing this type of trial, demonstrated in the differences between the ITT and PP analyses (doi:10.1056/NEJMe1601412). A lot was being asked of the early-introduction group families, with requests to give quite large quantities of foods not traditionally given to young British babies. And there is the possibility of bias by reverse causation: some potentially allergic babies may have instinctively resisted being given allergenic foods before having any reaction, hence their carers found it more difficult to give these and then deviated from the protocol. If official guidance changes to encourage early introduction of (at least) peanut and egg, which it probably should, then parents will need advice on how to prepare these foods and present them to 3-month-olds in a way that they will easily accept.

Competing interests None.

Provenance and peer review Commissioned; internally peer reviewed.

Accepted 11 July 2016

Published Online First 25 July 2016

Arch Dis Child 2016;101:802. doi:10.1136/archdischild-2016-311602

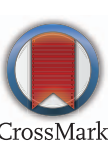

